\title{
Fraseo en español peninsular y modelo autosegmental y métrico
}

\author{
Phrasing in Peninsular Spanish and the Autosegmental Metrical model
}

\author{
Guillermo Toledo
}

Université Laval, Canadá y Conicet, Argentina.

e-mail: gatoledo@conicet.gov.ar

\begin{abstract}
Se analiza la influencia tonal provocada por el tono de frontera de la frase entonativa intermedia (H-) sobre el acento final de esa frase $\left(\mathrm{T}^{*}\right)$. La investigación se concreta con una metodología Autosegmental Metrical (AM). Se estudia un corpus integrado por 32 microdiscursos emitidos por un informador de Madrid, la lectura es formal. Los resultados indican que la presencia de $\mathrm{H}$ - en contacto con el último $\mathrm{T}^{*}$ causa cambios en la taxonomía tonal. Los paroxítonos y proparoxítonos se realizan con un retardo del pico, el primitivo es $\mathrm{L}^{*}+\mathrm{H}$ debido al tono creciente $\mathrm{H}-$ en la sílaba postónica. Los oxítonos no son afectados por $\mathrm{H}$ - debido a la posición final de $\mathrm{T}^{*}$. La asociación fonológica tiene lugar en la sílaba acentuada y se incrusta sobre el crecimiento de $\mathrm{H}-$. El primitivo es $\mathrm{L}+\mathrm{H}^{*}$. Estos resultados sugieren una asociación fonológica secundaria de último $\mathrm{T}^{*}$ con el tono de frontera $\mathrm{H}-$.
\end{abstract}

Palabras clave: fonología autosegmental y métrica, entonación española, asociación fonológica, asociación fonológica secundaria.

In this study the tonal influence provoked by the intermediate phrase edge rising tone $(\mathrm{H}-)$ on the preceding final accent $\left(T^{*}\right)$ is analyzed. The research is carried out with an Autosegmental Metrical (AM) methodology. A corpus composed of 32 micro-discourses produced by an informant from Madrid in formal fashion is studied. The results show that the presence of the $\mathrm{H}$ - in front of the last tonal accent causes changes in the tonal taxonomy. The paroxytone and proparoxytone $\mathrm{T}^{*}$ presents a peak delay, the primitive is $\mathrm{L}^{*}+\mathrm{H}$ due to the $\mathrm{H}$ - continuation rise on the post-tonic syllable. The oxytone $\mathrm{T}^{*}$ is not affected by the edge tone due to the final position of $\mathrm{T}^{*}$. The phonological association takes place on the accented syllable and overlaps the $\mathrm{H}$ - continuation rise. The primitive is $\mathrm{L}$ $+\mathrm{H}^{*}$. These results suggest a secondary phonological association of final $\mathrm{T}^{*}$ with the $\mathrm{H}$ - edge tone.

Key words: autosegmental and metrical phonology, spanish intonation, phonological association, secondary phonological association.

\section{INTRODUCCIÓN}

En el modelo autosegmental y métrico (AM) se proponen dos cadenas sintagmáticas, una segmental (el texto) y otra suprasegmental (el tono). Las dos cadenas son independientes, pero se asocian en ciertos puntos: las sílabas acentuadas con los tonos. Desde un punto de vista métrico, las sílabas acentuadas son fuertes. Los segmentos inacentuados de la cadena segmental son débiles. Los tonos son fuertes en las sílabas acentuadas. Los tonos en los segmentos inacentuados son transicionales: concatenan los acentos tonales al contorno de entonación. En cada acento tonal se actualizan uno o dos tonos: uno, central, alineado con la duración de la acentuada y otro, periférico, en la sílaba pretónica o en el inicio de la sílaba acentuada o en la sílaba postónica. Esa alineación con la sílaba acentuada se concreta en una asociación fonológica y en un primitivo fonológico: el acento tonal. Estos acentos tonales son targets 'blancos' estáticos, no son inflexiones dinámicas, y son relativos. Un tono relativamente bajo lo es con respecto a un rango tonal del hablante (el punto relativo más bajo o el punto relativo más alto). Esto significa que contrastan en forma paradigmática, según ese rango tonal y no en forma sintagmática de acuerdo al pico o al nivel tonal de otro acento adyacente (Beckman et al. 2005). En la secuencia LH (alto, bajo) en el desarrollo temporal de la sílaba acentuada se producen diferentes alineaciones fonéticas y, por consiguiente, también se producen diferentes acentos tonales. Los acentos tonales son prenucleares o nucleares. Los primeros se producen hasta la sílaba acentuada final de la frase, los segundos aparecen en la última sílaba acentuada antes de finalizar la frase. Para el español, la descripción estándar propone en el enunciado declarativo de foco ancho (todos los ítems nuevos) acentos prenucleares $\mathrm{L}^{*}+\mathrm{H}$ y un acento nuclear $\mathrm{L}+\mathrm{H}^{*}$. Los acentos prenucleares presentan un nivel $\mathrm{L}$ en la sílaba acentuada y un nivel $\mathrm{H}$ que se realiza en la sílaba postónica, el grado más alto de prominencia tonal se produce fuera de la duración de esa acentuada. 
Los acentos nucleares tienen un nivel L en el inicio de la sílaba acentuada o en la pretónica y un nivel $\mathrm{H}$ con una inflexión creciente hasta el final de la duración de la sílaba acentuada. El grado de prominencia tonal se produce en esa acentuada. El diacrítico estrella indica la localización temporal del grado de prominencia tonal en la sílaba acentuada: $\mathrm{L}^{*} \mathrm{o} \mathrm{H}^{*}$ (ver descripciones generales en Pierrehumbert 1980; Ladd 1996; Sosa 1999; Gussenhoven 2002, 2006; Hualde 2002, 2003; Beckman et al. 2002). La mayoría de los dialectos panhispánicos se comportan de este modo: acentos prenucleares $\mathrm{L}^{*}+\mathrm{H}$ y acentos nucleares $\mathrm{L}+\mathrm{H}^{*}$, los dos tonos fonemáticos están en contraste (Sosa 1999; Beckman et al. 2002). Sin embargo, en algunas lecturas fonológicas recientes hay divergencias (ver: Sección 2. 3).

Por otra parte, en niveles jerárquicos, se produce el intonation phrasing 'el fraseo entonativo' (Nespor y Vogel 1983, 1986). El enunciado declarativo consta de una frase entonativa final (frase mayor). Esa frase entonativa está integrada por frases entonativas intermedias (frase menor, frase fonológica $(\phi)$. Las frases entonativas intermedias están divididas en palabras fonológicas (w). Las palabras fonológicas están integradas por sílabas. Cada frase entonativa final tiene un tono de corte de frase entonativa final (L\%, H\%). Cada frase entonativa intermedia tiene un tono de corte de frase entonativa intermedia (L-, H). Cada frase entonativa intermedia tiene un acento nuclear, el acento tonal inmediatamente anterior al tono de corte de frase entonativa intermedia. Cada frase entonativa final tiene, de modo similar, un acento nuclear, el último acento tonal de la frase entonativa final y del enunciado (ver descripciones para el español en D'Imperio et al. 2004; Prieto 2006; Frota et al. (en proceso)). En suma, el esquema es el siguiente: La niña come pasteles. (La niña) $\phi$ (come pasteles) $\phi),((\mathrm{w}) \phi(\mathrm{w} w) \phi)$, La niña H- come pasteles L\%. El esquema estándar en español es (S) (VO) (ver un análisis sobre el español: Prieto 2006).

En este trabajo se analizan las frases intermedias con inflexión ascendente (H-) y la influencia ejercida sobre el último acento de esa frase. Se intenta demostrar un fenómeno fonológico de asociación secundaria entre los dos ítems: por la influencia del tono de frontera en la frase intermedia (H-), el acento final presenta una asociación fonológica in situ en los oxítonos, en la sílaba fuerte y acentuada $\left(\mathrm{H}^{*}\right)$, y los acentos finales paroxítonos y proparoxítonos muestran asociaciones desplazadas a la derecha, esto es, con tonos centrales débiles $\left(\mathrm{L}^{*}\right)$ en las acentuadas y tonos periféricos fuertes en las inacentuadas postónicas (H). El estudio se realiza en un corpus de microdiscursos leídos por un informante de Madrid. La estructura de este trabajo es la siguiente. En la Sección 2 se presenta la metodología general: la descripción de corpus estudiado (2.1); el análisis acústico realizado en las muestras (2.2); el modelo autosegmental y la taxonomía tonal resultante (2.3); la descripción de la frase entonativa intermedia (2.4). En la Sección 3 se analizan los casos de asociación secundaria entre el tono fuerte del acento tonal ( $\left.\mathrm{T}^{*}\right)$ y el tono de frontera de la frase entonativa intermedia $\mathrm{H}$-; se propone una taxonomía fonológica para los primitivos resultantes. En la Sección 4 se concluye sobre los resultados obtenidos y se argumenta sobre los hallazgos encontrados en este trabajo y confrontados con otras investigaciones anteriores; particularmente, los planteos que niegan la importancia prosódica del fraseo fonológico intermedio $(\phi)$, en español.

\section{METODOLOGÍA GENERAL}

2.1. Corpus. Los materiales analizados están constituidos por 32 microdiscursos. En ellos se estudian 44 frases entonativas intermedias $\mathrm{H}$-, con inflexión ascendente y continuidad semántica. A cada frase entonativa intermedia le corresponde un acento final que concluye esa frase. Según la taxonomía tonal propuesta en este trabajo, son acentos finales (también en Prieto 2006). Sin embargo, Sosa (1999: 56) considera acentos nucleares todos los acentos en posiciones finales de las frases entonativas, no existen frases entonativas intermedias en su planteo prosódico, en cambio, Herman (1998: 15) propone que todo acento tonal sea nuclear tanto en posición precedente a un tono de corte en frase entonativa intermedia como en frase entonativa final.

En el corpus se comunican instrucciones a los lectores de un texto de fonética y ortofonía del español (Moreno Fernández, 2000). Cada instrucción es leída por un único locutor peninsular, madrileño Las instrucciones aparecen antes de cada ejercicio. El informante produce lecturas con un estilo profesional. No hay repeticiones, aunque algunos ítems se reiteran en diferentes microdiscursos exhortativos. El símbolo IP indica la frase entonativa final; el símbolo $\phi$ indica frase entonativa intermedia. El símbolo L\% indica el tono de frontera de la frase entonativa final. El símbolo L- indica el tono de frontera de la frase entonativa intermedia, descendente. El símbolo $\mathrm{H}$ - indica el tono de frontera de la frase entonativa intermedia, ascendente. La pausa se marca por medio del símbolo \#. Los microdiscursos son de este tipo: Seis, nueve. (IP) L\% \# Escucha y repite los siguientes enunciados. (IP) L\% \# Oirás una vez ( $\phi)$ H- \# cada enunciado, ( $\phi)$ L- \# tendrás un tiempo para repetirlo $(\phi)$ H- \# y después $(\phi)$ H- \# 
volverás a oírlo ( $\phi$ ) H- \# antes de pasar al siguiente. (IP) L\% \# Comprueba que se pueden unir algunas vocales $(\phi) \mathrm{H}$ - pertenecientes a palabras distintas. (IP) L\% \#. Son emisiones con un valor pragmático similar: son exhortaciones con un énfasis ilocutivo producido con moderación y control. El corpus posee la continuidad discursiva de las producciones espontáneas y semiespontáneas y, además, las ventajas de los materiales no espontáneos: una segmentación precisa de las frases entonativas, en la mayoría de los casos, por medio de pausas claras tanto perceptivas como acústicas, tonos de corte en las frases entonativas intermedias perfectamente delimitados. Los acentos nucleares dentro de las frases intermedias tienen, en muchos casos, consonantes sonantes en el ataque de la sílaba tónica y postónica para asegurar una lectura indudable de los fenómenos de realización in situ o posrealización del pico tonal (ver un análisis global del corpus (acentos prenucleares, acentos nucleares, tonos de corte en frases entonativas intermedias (H-, L-) y en frases entonativa finales (H\%, L\%) en Toledo 2006 a)).

2.2. Análisis acústico. Se calculan los contornos de entonación en los microdiscursos seleccionados. Para ese fin, se utiliza el analizador acústico Speech Analyzer, versión 2.7 (Summer Institute of Linguistics, 2006). Se obtienen datos en semitonos. Asimismo, se observan los contornos de intensidad que permiten la visualización de la envolvente de cada sílaba y sus fronteras, esto ayuda a la decisión del punto de mayor prominencia tonal. En los casos con conflicto en el contorno, se siguen estos criterios: en las sílabas con contornos planos u ondulantes se alinea el contorno de la fundamental con el contorno de intensidad. El valor medido es el punto de consonancia de los dos contornos. Los tonos en las sílabas con pulsos o bandas de ruido en el ataque (oclusivas, fricativas y africadas) se miden en el estado estable de la vocal, el tono inmediato a la transición entre la consonante en el ataque y la vocal siguiente (CV o CCV) es espurio debido a la influencia de la intensidad del ruido sobre el valor tonal. Los gráficos se realizan por medio del programa de análisis ya indicado. La zona superior de esas Figuras registra la forma de onda segmentada en sílabas (entre barras verticales) y la transcripción ortográfica de esas sílabas. La zona inferior muestra el contorno de entonación también segmentado en sílabas (entre barras verticales). Esta zona está segmentada en semitonos (st.), en bandas de 2 st. (rejilla horizontal, es estándar en el programa de computación).

2.3. El modelo autosegmental: taxonomías resultantes. Hualde (2002) critica algunas lecturas fonológicas de acentos tonales. El primitivo fonológico $\mathrm{L}^{*}+\mathrm{H}$ se describe del siguiente modo: en superficie, existe un valle $\left(\mathrm{L}^{*}\right)$ en el inicio de la sílaba acentuada, un crecimiento de la frecuencia fundamental en el desarrollo temporal de esa sílaba acentuada y un pico de la fundamental que supera los límites de la acentuada y ocupa el espacio de la sílaba postónica (H). Garrido et al. (1993), Llisterri et al. (1995) y de la Mota $(1997,2005)$ observan que este tipo de acento, $\mathrm{L}^{*}+\mathrm{H}$, tiene una frecuencia de aparición absoluta, que es generalizada para el español. Face (2001) también indica un tono similar para los acentos tonales del pretonema, esto es, todos los acentos, excepto el final. Willis (2003) también se suma a los estudios precedentes. Observa la presencia constante de acentos tonales $\mathrm{L}^{*}+\mathrm{H}$ en corpus del dialecto de la República Dominicana.

Prieto (1998), Prieto y Shih (1995), Prieto et al. (1995), Prieto et al. (1996) y Prieto (2001) analizan la alineación tonal, fonética, y la asociación fonológica en corpus de español mexicano. Desde el plano fonético, observan un crecimiento tonal en la sílaba acentuada y el pico se produce casi regularmente en la sílaba postónica. Desde el punto de vista fonológico, estos crecimientos tonales se asocian con el tono $\mathrm{H}^{*}$. El pico se vincula con la sílaba acentuada, ya sea con el anclaje dentro de la sílaba acentuada o en otro punto de la sílaba postónica. En esa secuencia LH, el nivel del valle tonal (L) coincide con el inicio de la sílaba acentuada, se indica en la superficie fonética, pero no se registra en la asociación fonológica. Igualmente, en su tesis doctoral, Nibert (2000) rechaza un tono $\mathrm{L}^{*}+\mathrm{H}$ para las actualizaciones de superficie con desplazamientos a la derecha, esto es, desplazado a la sílaba postónica. Proponen en las seis investigaciones un solo primitivo fonológico $\mathrm{H}^{*}$ como predominante del español, ya sea con desplazamiento hacia a la derecha o sin desplazamiento, en este caso, con el pico en algún punto de la sílaba acentuada. Hualde (2002) se opone a esta lectura tonal, observa que el nivel tonal L en el inicio de la sílaba acentuada es un factor de superficie que debe ser parte de una lectura fonológica. Para recuperar este nivel de superficie, el valle en el inicio de la sílaba acentuada, presenta un primitivo $(\mathrm{L}+\mathrm{H}) *$ para los acentos tonales del español. El paréntesis es un recurso fonológico con el fin de recuperar la asociación de los dos tonos dentro de la secuencia LH en el interior de la sílaba acentuada. El nivel L aparece en el inicio de la sílaba acentuada y el nivel $\mathrm{H}$ (el pico en superficie) puede actualizarse en la sílaba acentuada o en la sílaba postónica. Face (2001) también coincide con este tipo de análisis fonológico. Alvord (2006) propone una taxonomía tonal de acentos prenucleares $\mathrm{L} *+\mathrm{H}$ en el español hablado en la ciudad de Miami. Este tipo de acento se percibe como una tonalidad baja; en contraste, el 
acento nuclear $\mathrm{L}+\mathrm{H}^{*}$ se percibe con una tonalidad alta.

Colantoni y Gurlekian (2004) y Colantoni (2005) analizan los acentos prenucleares en corpus de español, en el dialecto de Argentina. Sus hallazgos indican acentos $\mathrm{H}^{*}+\mathrm{L}$ con una frecuencia de aparición relativa de $90,74 \%$ y acentos $\mathrm{L}+\mathrm{H}^{*}$ con una frecuencia de aparición relativa de $1,85 \%$. Los fenómenos de posrealización del pico sobre la sílaba postónica son rarísimos en este corpus. Barjam (2004) obtiene en el español de Buenos Aires una taxonomía compuesta por dos tonos fonemáticos con la asociación fonológica dentro de la sílaba acentuada: $\mathrm{L}+\mathrm{H}^{*} \mathrm{y} \mathrm{L}+{ }^{\wedge} \mathrm{H}^{*}$. El diacrítico ${ }^{\wedge}$ indica un upstep 'escalonamiento creciente'. El primer acento fonemático aparece en posiciones prenucleares. El segundo acento fonemático se realiza en posiciones nucleares. En sus hallazgos, el tono se ancla siempre a la sílaba acentuada. Los resultados coinciden, en líneas generales, con los de Toledo (2000, 2001 b).

Toledo $(2000,2001 \mathrm{~b})$ observa un pretonema con realizaciones acentuales $\mathrm{H}^{*}+\mathrm{L}$. Encuentra una mayor frecuencia de acentos $\mathrm{H}^{*}+\mathrm{L}$ en los corpus de habla espontánea; esto significa que en materiales discursivos se propician los contrastes entre el acento $\mathrm{H}^{*}$ y la sílaba postónica L. En habla de laboratorio, el tiempo del pico es interno a la duración de la sílaba acentuada, $\mathrm{H}^{*}$; sobre todo en acentos prenucleares fuera de la primera posición del pretonema. Asimismo, Toledo (2002, 2004 a) obtiene resultados en discursos del corpus CREA (Universidad Autónoma de Madrid y Real Academia Española). Se observa la prominencia en la sílaba tónica tanto en acentos monotonales como bitonales. Toledo (2003) analiza discursos del corpus DIES (Universidad de Alcalá de Henares y Real Academia Española) que confirman nuevamente que la prominencia tonal se realiza dentro del desarrollo temporal de la sílaba acentuada, esto es, un primitivo fonológico $\mathrm{H}^{*}$. En el español de Cuba, Toledo (2004 b) obtiene resultados en tres discursos radiofónicos emitidos por hablantes, que revelan una realización relevante de la prominencia más alta en la sílaba acentuada, acentos monotonales $\mathrm{H}^{*}$ y combinados con tonos bajos, a izquierda y/o a derecha. Toledo (2005) presenta resultados obtenidos por medio del análisis de la entonación en cuatro discursos radiofónicos emitidos por cuatro informantes de español de Tenerife, Islas Canarias, que indican dos tendencias tonales registradas en estudios ya presentados. Primero, el estudio de los pretonemas muestra una variedad de acentos tonales: $\mathrm{H}^{*}, \mathrm{~L}+\mathrm{H}^{*}, \mathrm{H}^{*}+\mathrm{L}$. Segundo, esos acentos tonales presentan la prominencia en $\mathrm{H}^{*}$ con una frecuencia de aparición relativa muy alta, entre $81,35 \%$ y $87,69 \%$. Toledo (2006 a) analiza microdiscursos peninsulares integrados por pretonemas, tonos de corte en frases entonativas intermedias, acentos nucleares y tonos de corte en frases entonativas finales. Los resultados muestran que los picos de F0 se actualizan dentro de la sílaba acentuada. En los casos en que existen efectos de posrealización del pico, los dos tonos tienen similares prominencias, sin diferencias perceptivas en contraste. Estos hallazgos coinciden, en líneas generales, con lo propuesto por Toledo (2006 a, 2006 b, en prensa a, en prensa b). Sin embargo, Fernández Planas et al. (2002) obtienen acentos prenucleares $\mathrm{L}^{*}+\mathrm{H}$, aunque la taxonomía tiene variedad de tonos. En este caso, los corpus de laboratorio, oraciones enunciativas, pertenecen a producciones emitidas por hablantes de español de Barcelona y de Alicante. Del mismo modo, Toledo (2006 b, en prensa b) logra hallazgos similares en corpus de oraciones enunciativas emitidos por una hablante de Barcelona. Esto sugiere que la posrealización del tono en la sílaba postónica, ya sea con o sin contraste perceptivo, no es una tendencia general del español. Es un fenómeno sumamente complejo debido a un número enorme de influencias, entre otras, por el tipo de acento (paroxítono, oxítono, proparoxítono) (Prieto y Torreira, en prensa) y la tensión producida por los tonos de corte en las fronteras del fraseo prosódico (Toledo y Ramírez Verdugo (en proceso).

2.4. Características de la frase entonativa intermedia. Prieto (2006) analiza los patrones fonéticos y la fonología de la frase intermedia. Observa que el acento tonal final de la frase tiene mayor prominencia que los restantes acentos tonales que la constituyen. En general, esta frase se produce entre pausas. Tiene un tono de frontera (H- o L-). Particularmente, la frase intermedia H- presenta un crecimiento tonal en la última sílaba de la palabra fonológica final, que se produce sobre el alargamiento temporal final producido en esa sílaba.

Por otra parte, hay también diferencias semánticas y pragmáticas, esto es, discursivas entre los dos tipos de frases intermedias. La frase intermedia con tono de frontera L- indica la separación entre esa frase y la siguiente. En cambio, la frase intermedia con tono de frontera $\mathrm{H}$ - indica que esa frase tiene cohesión semántica con la siguiente frase (ver: Pierrehumbert y Hirschberg 1986, 1990; Prevost 1995: $48)$.

\section{ANÁLISIS DE ASOCIACIONES FONOLÓGICAS SECUNDARIAS EN LOS MICRODISCURSOS}


En la figura 1 se indica la asociación fonológica secundaria entre el acento tonal final y el tono de frontera $\mathrm{H}$-. El acento tonal final es paroxítono. La frase entonativa intermedia es parte del siguiente microdiscurso: Dos, dos. (IP) L\% \# Pronuncia el nombre de las letras ( $\phi$ ) L- \# que aparecen a continuación. (IP) L\% \# Después, ( $\phi)$ H- \# repite letra por letra $(\phi)$ H- \# escucha la grabación $(\phi)$ H- \# y compara. (IP) L\% \#.

En la figura 1 se observa claramente que el acento final pierde su asociación primaria con la sílaba acentuada debido a la influencia del tono de frontera H-. La sílaba postónica está incrustada dentro del tono de frontera y cambia su asociación fonológica. El primitivo fonológico es $\mathrm{L}^{*}+\mathrm{H}$. En el caso del acento tonal anterior al acento final, la asociación fonológica se produce con la sílaba acentuada, el primitivo fonológico es $\mathrm{H}^{*}+\mathrm{L}$. Este fenómeno fonológico es privativo de los acentos tonales paroxítonos cuando se produce una asociación fonológica, secundaria, con el tono de frontera H-.

En la figura 2 se muestran dos frases frases entonativas intermedias con los tonos de frontera H-. Las frases entonativas pertenecen al microdiscurso siguiente: Dos, tres. (IP) L\% \# Escucha el nombre en singular y en plural ( $\phi$ ) H- \# de varias letras. (IP) L\% \# Repítelos después de cada una de ellas. (IP) L\% \# Por ejemplo, ( $\phi) \mathrm{H}$ - \# la hache, $(\phi) \mathrm{H}$ - \# las haches. (IP) L\% \#. Las dos frases entonativas intermedias, con acentos tonales paroxítonos (por ejemplo y la hache) presentan efectos prosódicos de asociación fonológica secundaria con los respectivos tonos de frontera H-. Naturalmente, el efecto no se produce en el acento tonal de la frase entonativa final, las haches frente a $\mathrm{L} \%$.

En la figura 3 se observa la taxonomía fonológica resultante en detalle debido a la asociación fonológica secundaria entre el acento tonal final (el único en este caso: Por ejemplo) de la frase entonativa intermedia y el tono de frontera $\mathrm{H}-$. El acento tonal es $\mathrm{L}^{*}+\mathrm{H}$ : el tono bajo se asocia a la sílaba acentuada y la sílaba postónica, alta, se asocia con el tono de frontera $\mathrm{H}$ -

En la figura 4 se observan dos acentos tonales finales frente a los tonos de frontera $\mathrm{H}$-. Las dos frases intermedias pertenecen a este microdiscurso: Ocho, nueve. (IP) L\% \# Escucha y repite cada enunciado. (IP) L\% \# Compara tu entonación ( $\phi) \mathrm{H}$ - \# con la de la grabación, $(\phi) \mathrm{H}-$ \# e intenta imitarla. (IP) L\% \#. Se marcan con letra negrita ( $\phi)$ H- \# la primera sílaba tónica (IP) L\% \#. La asociación fonológica entre los acentos finales de las frases entonativas intermedias y las respectivas sílabas acentuadas es primaria debido a la posición del acento tonal en los oxítonos. Asimismo, la asociación fonológica es secundaria: se produce entre los acentos tonales oxítonos finales y los tonos de frontera de las frases entonativas intermedias $\mathrm{H}-$. Se estudian en detalle en la figura 5.

En la figura 5 se observan en detalle dos acentos tonales oxítonos finales frente a los tonos de frontera de frases entonativas intermedias H-. Se indican los primitivos fonológicos resultantes, en los dos casos son tonos $\mathrm{L}+\mathrm{H}^{*}$. La asociación es primaria, con la sílaba acentuada, pero es también secundaria, con el tono de frontera H-. La transcripción por tonos (parte superior de la Figura) indica la tendencia ascendente o descendente del fraseo.

En la figura 6 se muestran dos acentos tonales oxítonos, uno es final y frente al tono de frontera de frase entonativa intermedia. Esta frase entonativa es parte del microdiscurso ya explicado en detalle (arriba: texto de la figura 2). Se produce una asociación primaria entre el tono y la sílaba acentuada y una asociación secundaria con el tono de frontera H-. Este tono se incrusta en la sílaba acentuada y no varía la asociación fonológica del acento tonal, es $\mathrm{L}+\mathrm{H}^{*}$. Los dos primitivos fonológicos tienen una alineación fonética similar y una asociación fonológica también similar. Naturalmente, el acento tonal final tiene doble asociación: una con la sílaba acentuada y una, secundaria, con el tono de frontera H-.

En la figura 7 se observa un acento tonal final proparoxítono frente a un tono de frontera en una frase entonativa intermedia. El acento tonal es tónica y el tono de frontera es H-. El microdiscurso es el que sigue: Siete, cinco. (IP) L\% \# Escucha y subraya ( $\phi) \mathrm{H}$ - las sílabas tónicas ( $\phi$ ) H- de las siguienes palabras. (IP) L\% \#. Después de las dos frases entonativas intermedias se produce un fenómeno tonal de resetting 'restablecimiento' de la curva de entonación. Después de la primera frase entonativa intermedia (Escucha y subraya) el restablecimiento de la entonación es total, los valores son muy cercanos al primer pico de la frase entonativa anterior. Luego de la segunda frase entonativa intermedia (las sílabas tónicas), el restablecimiento es parcial y escalonado. El acento tonal final paroxítono sufre un efecto de asociación secundaria frente al tono de frontera de la frase entonativa intermedia. Por este efecto, la sílaba acentuada es baja y la sílaba postónica es alta. El primitivo fonológico es $\mathrm{L}^{*}+\mathrm{H}$. Asimismo, se produce un patrón entonativo similar en el acento tonal final paroxítono asociado secundariamente con el tono de frontera de la frase entonativa intermedia (ver casos similares en las figuras 1, 2 y 3). Así, Escucha y subraya es $\left[\mathrm{w}\left(\mathrm{L}+\mathrm{H}^{*}\right) \mathrm{w}\left(\mathrm{L}^{*}+\mathrm{H}\right)\right] \phi \mathrm{H}$-. El símbolo w (ya explicado: 2.1) indica las dos palabras fonológicas de la frase entonativa intermedia, un nivel prosódico inferior con referencia a $\phi$.

En la figura 8 se muestra un acento nuclear proparoxítono dentro de una frase entonativa final. Esa frase entonativa integra el microdiscurso Siete, dos. (IP) L\% \# Escucha ( $\phi) \mathrm{H}$ - y subraya ( $\phi) \mathrm{H}-$ las 
sílabas tónicas (IP) L\% \#. El acento nuclear (tónicas) se enfrenta, en este caso, a un tono de frontera L\%. La asociación fonológica es primaria con la sílaba acentuada y es secundaria con el tono de frontera. La sílaba tónica mantiene su prominencia y la sílaba postónica se incrusta en el tono de frontera L\%. El primitivo fonológico es $\mathrm{H}^{*}+\mathrm{L}$ (observar el tono $\mathrm{L}^{*}+\mathrm{H}$ en la figura 7 ).

En la figura 9 se puede observar una acento tonal proparoxítono final de frase entonativa intermedia y el tono de frontera de esa frase H-. La transcripción por tonos se explica en el texto de la Figura 5. Es parte del microdiscurso siguiente: Siete, trece. (IP) L\% \# Escucha las palabras siguientes, $(\phi) \mathrm{H}-$ \# subraya la sílaba tónica ( $\phi)$ L- \# y coloca el acento gráfico $(\phi)$ H- cuando sea necesario (IP) L\% \# Tendrás un tiempo para subrayar $(\phi)$ H- \# y escribir el acento gráfico (IP) L\% \#. El acento tonal proparoxítono final de frase entonativa intermedia (gráfico) presenta una asociación fonológica secundaria con el tono de frontera $\mathrm{H}-$. El tono es bajo en la sílaba acentuada y alto en la sílaba postónica debido al solapamiento de esa sílaba con el tono de frontera $\mathrm{H}-$. El acento tonal es, entonces, $\mathrm{L}^{*}+\mathrm{H}$.

En la figura 10 se muestra el comportamiento tonal de un acento nuclear (gráfico) frente a un tono de frontera de frase entonativa final $\mathrm{L} \%$. El tono alto se asocia a la sílaba acentuada y el tono bajo lo hace con la sílaba postónica. El acento tonal resultante es $\mathrm{H}^{*}+\mathrm{L}$. La asociación primaria del tono se produce con la sílaba acentuada. La asociación secundaria de la sílaba postónica se produce con el tono de frontera.

\section{CONCLUSIONES Y DISCUSIÓN}

El análisis de los acentos finales de frase entonativa intermedia con tonos de frontera $\mathrm{H}$ - muestra una asociación fonológica primaria, estándar. Las sílabas acentuadas, fuertes, se asocian fonológicamente con el tono en forma primaria. Asimismo, presenta una asociación fonológica secundaria con los tonos de frontera. Esta asociación fonológica secundaria varía según el tipo de acento final. Los acentos finales paroxítonos presentan una alineación fonética desplazada a la sílaba postónica debido a la incrustación de esa postónica en el tono de frontera H-. La asociación fonológica es secundaria, y los acentos tonales finales paroxítonos son, preferentemente, $\mathrm{L}^{*}+\mathrm{H}$ : la sílaba acentuada es baja y la sílaba postónica es $\mathrm{H}$. Es decir, la sílaba acentuada presenta un tono L y en diferentes combinaciones tonales. En cambio, los acentos finales oxítonos presentan una alineación fonética in situ. Por ello, la sílaba acentuada se asocia fonológicamente con el tono en forma primaria, pero se asocia también con el tono de frontera, en este caso en forma secundaria. Los acentos tonales finales son, preferentemente, $\mathrm{L}+\mathrm{H}^{*}$. En todo caso, la sílaba acentuada presenta un tono $\mathrm{H}$ y en diferentes combinaciones tonales.

Los acentos finales proparoxítonos muestran un esquema entonativo similar a los acentos finales paroxítonos. La influencia de los tonos de frontera $\mathrm{H}$ - producen sílabas postónicas con tonos $\mathrm{H}$ y sílabas acentuadas con tonos L. Se produce un escalonamiento ascendente entre las sílabas y desde la acentuada. Los acentos tonales finales proparoxítonos son, preferentemente, $\mathrm{L}^{*}+\mathrm{H}$. El fenómeno inverso se produce con tonos de frontera de frase entonativa intermedia L- y con tonos de frontera de frase entonativa final L\%. Obviamente, la asociación fonológica secundaria con estos tonos de frontera influye en el comportamiento tonal de la sílaba postónica. Por ello, los acentos tonales frente a este tipo de tonos de frontera es, preferentemente, $\mathrm{H}^{*}+\mathrm{L}$.

Este estudio confirma el argumento de asociación fonológica secundaria propuesto para otras lenguas (Grice 2006; Grice et al. 2000; Grice y Baumann 2006). Asimismo, confirma la importancia de la frase entonativa intermedia en español (Nibert 2000; Hualde 2002; Prieto 2006). En cambio, no confirma el análisis propuesto por Sosa (1999), Beckman et al. (2002) y Jun (2005) cuando no consideran la frase entonativa intermedia como un nivel jerárquico entre la palabra fonológica (w) y la frase entonativa mayor (IP). El proceso fonológico de asociación secundaria en la frontera derecha de la frase reafirma esta unidad para el español, ya estudiada en corpus de laboratorio tanto por Prieto (2006), en corpus peninsulares (Madrid y Burgos) como por Toledo y Ramírez Verdugo (en proceso), en dialectos de español de Buenos Aires y de Barcelona.

\section{OBRAS CITADAS}

Alvord, Scott. 2006. Spanish Intonation in Contact: the Case of Miami Cuban Bilinguals. Tesis doctoral. Minnesota: University of Minnesota.

Barjam, John Patrick. 2004. The Intonational Phonology of Porteño Spanish. Tesis de Maestría. Los Angeles: University of California at Los Angeles. 
Beckman, Mary E., et al. 2002. "Intonation across Spanish, in the tones and break indices framework". Probus 14: 9-36.

2005. Julia Hirschberg y Stefanie Shattuck-Hufnagel. "The original ToBI system and the evolution of the ToBI framework". Sun-Ah Jun (ed.) Prosodic Typology: The Phonology of Intonation and Phrasing. Reino Unido y Europa: Oxford University Press, en www.ling.ohio-state.edu/ tobi/JunBook/BeckHirschShattuck ToBI.pdf.

Colantoni, Laura. 2005. "Peak alignment of pre-nuclear and nuclear accents in Argentine Spanish", $2^{\text {nd }}$ Spanish ToBI Workshop, PaPI 2005 Phonetics and Phonology in Iberia. Barcelona: Universidad Autónoma de Barcelona, en www.seneca.uab.es /filologiacatalana/papi/tobi/Colantoni.ppt, 22 de junio de 2005.

_ y Jorge Gurlekian. 2004. "Early peak alignment and deep fall in Buenos Aires broad focus declaratives". 9th Conference on Laboratory Phonology. Illinois: University of Illinois at Urbana-Champaign, en www.linguist.jussieu.fr / marandin/pdf/cours2.pdf, 24-25 de junio de 2004.

De la Mota, Carme. 1997. "Prosody of sentences with contrastive new information in Spanish", en Antonis Botinis (ed.) Intonation: theory, models and applications, Proceedings of an ESCA Workshop: 75-78. Grenoble: European Speech Communication Association.

- 2005. "Alignment, word boundaries and speech rate in Castilian Spanish", PaPI 2005 Phonetics and Phonology in Iberia. Barcelona: Universidad Autónoma de Barcelona, 20 de junio de 2005.

D'Imperio, Mariapaola et al. 2004. "Intonational phrasing in Romance: syntactic and prosodic structure”. Sónia Frota, Marina Vigário, María Joao Freitas (eds.) Prosodies: 59-98. The Hague: Mouton de Gruyter.

Face, Timothy. 2001. Intonational Marking of Contrastive Focus in Madrid Spanish. Tesis doctoral. Ohio: The Ohio State University.

Fernández Planas, Ana María et al. 2002. “Taxonomía autosegmental en la entonación del español peninsular”. Jesús Díaz García (ed.) Actas del II Congreso de Fonética Experimental: 180-186. Sevilla: Universidad de Sevilla.

Frota, Sónia et al. 2007. "The phonetics and phonology of intonational phrasing in Romance". Pilar Prieto, Joan Mascaró y María-José Solé (eds.) Laboratory Approaches to Romance Phonology. Amsterdam y Filadelfia: John Benjamins (en proceso).

Garrido, Juan M. et al. 1993. "Prosodic differences in reading style: isolated vs. contextualized sentences". Eurospeech 93: 573-576.

Grice, Martine. 2006. "Intonation”. Keith Brown (ed.) Encyclopedia of Language and Linguistics, 2da. Edición, vol. 5: 778-788. Oxford: Elsevier.

— Robert D. Ladd y Amalia Arvaniti. 2000. "On the place of phrase accents in intonational phonology". Phonology 17: 143-185.

y Stefan Baumann. 2006. "An intoduction to intonation - Functions and models": 1-21, en Mitarbeiter/mgrice/grice/publications/Publications.html.

Gussenhoven, Carlos. 2002. "Phonology of intonation”. Glot International 6, 9-10; 271-284.

—. 2006. "The phonology of intonation", en www.let.ru.nl/gep/carlos/ Gussenhoven HandbookPauldeLacy.

Herman, Rebecca. 1998. Intonation and Discourse Structure in English: Phonological and Phonetic Markers of Local and Global Discourse Structure. Tesis doctoral. Ohio: The Ohio State University, en ling.ohiostate.edu/publications/pdf/Herman.pdf.

Hualde, José Ignacio. 2002. "Intonation in Spanish and the other Ibero-Romance languages: overview and status quaestionis". Caroline Wiltshire y Joaquim Camps (eds.), Romance Phonology and Variation, Selected Papers from the $30^{\text {th }}$ Linguistic Symposium on Romance Languages: 101-116. Amsterdam: John Benjamins.

— . 2003. "El modelo métrico y autosegmental”. Pilar Prieto (ed.) Teorías de la entonación. Barcelona: Editorial Ariel.155-184.

Jun, Sun-Ah. 2005. "Prosodic typology". Sun-Ah Jun (ed.) Prosodic Typology: The Phonology of Intonation and Phrasing: 430-457. Reino Unido y Europa: Oxford University Press.

Ladd, Robert D. 1996. Intonational Phonology. Cambridge: Cambridge University Press.

Llisterri, Joaquim, et al. 1995. "Factors affecting F0 peak displacement in Spanish". ESCA, Eurospeech'95, 4th Conference on Speech Communication and Technology: 2061-2064.

Moreno Fernández, Francisco. 2000. Ejercicios de fonética española para hablantes de inglés. Madrid: Arco/Libros S. L.

Nespor, Marina e Irene Vogel. 1983. "Prosodic structure above the word". Anne Cutler y Robert D. Ladd (eds.) Prosody: Models and Measurements: 123-140. Berlín, Heidelberg, Nueva York y Tokio: Springer-Verlag.

- 1986. Prosodic Phonology. Dordrecht: Foris.

Nibert, Holly. 2000. Phonetic and phonological evidence for intermediate phrasing in Spanish intonation. Tesis doctoral. Illinois, Urbana Champaign: University of Illinois at Urbana-Champaign.

Pierrehumbert, Janet. 1980. The Phonology and Phonetics of English Intonation. Tesis doctoral. Cambridge, Massachusetts: The MIT Press. 
y Julia Hirschberg. 1986. "The intonational structuring of discourse". Proceedings of the $24^{\text {th }}$ Annual Meeting of the Association for Computational Linguistics, en citeseer.ist.psu.edu/julia86intonational.html.

y Julia Hirschberg. 1990. "The meaning of intonational contours in the interpretation of discourse". Philip R. Cohen, Jerry Morgan y Martha E. Pollock (eds.) Intentions in Communication: 271-312. Cambridge, MA: the MIT Press.

Prevost, Scott Allan. 1995. A Semantic of Contrast and Information Structure for Specifying Intonation in Spoken Language Generation. Tesis doctoral: Pensilvania: University of Pennsylvania.

Prieto, Pilar. 1998. "The scaling of the L values in Spanish downstepping contours". Journal of Phonetics 26: 261282.

—. 2001. Review of Sosa, Juan Manuel (1999): “La entonación del español”, Madrid: Cátedra. Linguistics: 39-46.

—. 2006. "Phonological phrasing in Spanish". Sonia Colina y Fernando Martínez-Gil (eds.) Optimality-Theoretic Advances in Spanish Phonology: 39-60. Amsterdam y Filadelfia: John Benjamins.

_ y Chilin Shih. 1995. "Effects of tonal clash on downstepped H* accents in Spanish". EUROSPEECH '95: 1307-1310.

__ Jan van Santen y Julia Hirschberg. 1995. "Tonal alignment patterns in Spanish". Journal of Phonetics 23: 429-451.

— Chilin Shih y Holly Nibert. 1996. "Pitch downtrend in Spanish". Journal of Phonetics 24: 445-473.

_ y Francisco Torreira. 2007. "The segmental anchoring hypothesis revisited. Syllable structure and speech rate effects on peak timing in Spanish". Journal of Phonetics (en prensa).

Sosa, Juan. 1999. La entonación del español: Su estructura fónica, variabilidad y dialectología. Madrid: Cátedra.

Toledo, Guillermo. 2000. "H en el español de Buenos Aires". Langues et Linguistique 26: 107-127.

—. 2001 b. "Taxonomía tonal en español”. Language Design 3: 1-20.

2002. "Acentos tonales en discursos". Jesús Díaz García (ed.) Actas del I/ Congreso de Fonética Experimental: 78-88. Sevilla: Universidad de Sevilla.

—. 2003. "Modelo autosegmental y entonación: los corpus DIES-RTVP". Estudios de Fonética Experimental XII: $143-163$.

—. 2004 a. "Modelo autosegmental y entonación: una muestra del corpus CREA". Revista de Filología 22 (RFULL): 313-327.

— 2004 b. "Prominencia H*: una muestra de español de Cuba". Estudios de Fonética Experimental XIII: 181202.

—. 2005. "Modelo autosegmental y dialecto: el español de Tenerife". Johannes Kabatek (Ed.) Revista Internacional de Lingüística Iberoamericana, Volumen III, Número 2 (6) (Volumen sobre Aspectos prosódicos de las lenguas iberorrománicas): 67-83.

—. 2006 a. "Tonos estrellados: Una argumentación". Estudios de Fonética Experimental XV: 99-131.

— 2006 b. "Tiempo tonal en dos contextos y en dos dialectos". Revista de Filología (RFULL) 24: 253-268.

—. 2007. "Alineación tonal en español", Actas del III Congreso de Fonética Experimental, Santiago de Compostela, 24-26 octubre de 2005 (en prensa a).

- 2007 "Choque tonal en español", Actas del III Congreso de Fonética Experimental, Santiago de Compostela, 24-26 octubre de 2005 (en prensa b).

— y María Dolores Ramírez Verdugo. 2007. "Spanish phrasing: Secondary association of $\mathrm{T}^{*}$ in face of intermediate phrase edge tone H- (laboratory data)". Phonetics and Phonology in Iberia (PaPI) 2007, Universidad de Minho, Braga, Portugal, 25-26 de junio de 2007 (en proceso).

Willis, Erik. 2003. The intonational system of Dominican Spanish: findings and analysis. Tesis doctoral. Illinois, Urbana-Champaign: University of Illinois Urbana-Champaign. 